Erratum

\title{
Erratum: Yu, W.; Jiao, J. Sustainability of Abandoned Slopes in the Hill and Gully Loess Plateau Region Considering Deep Soil Water. Sustainability 2018, 10,2287
}

\author{
Weijie Yu ${ }^{1}$ and Juying Jiao ${ }^{1,2, *}$ \\ 1 State Key Laboratory of Soil Erosion and Dry Land Farming on the Loess Plateau, Institute of Soil and Water \\ Conservation, Northwest A\&F University, Yangling 712100, China; yuweij@nwsuaf.edu.cn \\ 2 Institute of Soil and Water Conservation, Chinese Academy of Sciences and Ministry of Water Resources, \\ Yangling 712100, China \\ * Correspondence: jyjiao@ms.iswc.ac.cn; Tel.: +86-1347-437-5827
}

Received: 12 April 2019; Accepted: 15 April 2019; Published: 18 April 2019

The authors did not notice during the proofreading phase that there were errors in the affiliations, funding, and acknowledgments. The authors wish to make the following corrections to this paper [1]:

(1) The affiliations were originally:

1 State Key Laboratory of Soil Erosion and Dry Land Farming on the Loess Plateau, Institute of Soil and Water Conservation, Northwest A\&F University, Yangling 712100, China; yuweij@nwsuaf.edu.cn

2 Institute of Soil and Water Conservation, Chinese Academy of Sciences and Ministry of Water Resources, Yangling 712100, China

The affiliations should be replaced with:

1 State Key Laboratory of Soil Erosion and Dry Land Farming on the Loess Plateau, Institute of Soil and Water Conservation, Northwest A\&F University, Yangling 712100, China; yuweij@nwsuaf.edu.cn

2 State Key Laboratory of Soil Erosion and Dry Land Farming on the Loess Plateau, Institute of Soil and Water Conservation, Chinese Academy of Sciences and Ministry of Water Resources, Yangling 712100, China

(2) The funding was originally:

The National Key Research and Development Program of China (No. 2016YFC0501604) and the National Science Foundation of China (No. 41771322; 41371280).

The funding should be replaced with:

The National Key Research and Development Program of China (No. 2016YFC0501604) and the National Science Foundation of China (No. 41771319; 41371280).

(3) The acknowledgments were originally:

This study was supported by the National Key Research and Development Program of China (No. 2016YFC0501604) and the National Science Foundation of China (No. 41771322; 41371280). The acknowledgments should be replaced with:

This study was supported by the National Key Research and Development Program of China (No. 2016YFC0501604) and the National Science Foundation of China (No. 41771319; 41371280). 
The authors would like to apologize for any inconvenience caused to the readers by these changes. The manuscript will be updated, and the original copy will remain online on the article webpage.

\section{Reference}

1. Yu, W.; Jiao, J. Sustainability of Abandoned Slopes in the Hill and Gully Loess Plateau Region Considering Deep Soil Water. Sustainability 2018, 10, 2287. [CrossRef]

(c) 